\section{Toxic epidermic necrolysis by allopurinol: a case report}

\author{
Rosa Paola Cerra, ${ }^{1}$ Alberto Castagna, ${ }^{2}$ \\ Laura Greco, ${ }^{1}$ Rosaria Anna Galea, ${ }^{1}$ \\ Maria Lucia Citraro, ${ }^{4}$ \\ Carmen Ruberto, ${ }^{2}$ \\ Giuseppe Coppolino, ${ }^{3,4}$ \\ Giovanni Ruotolo ${ }^{1}$ \\ ${ }^{1}$ Geriatric Unit, Pugliese-Ciaccio \\ General Hospital, Catanzaro; ${ }^{2}$ Centre \\ for Cognitive Disorders and Dementia, \\ Azienda Sanitaria Provinciale di \\ Catanzaro, Catanzaro; ${ }^{3}$ Renal Unit, \\ Department of Health Sciences, Magna \\ Graecia University, Catanzaro; \\ ${ }^{4}$ Nephrology and Dialysis Unit, \\ Pugliese-Ciaccio General Hospital, \\ Catanzaro, Italy
}

\section{Abstract}

Toxic epidermal necrolysis (TEN) or Lyell's syndrome is a rare but serious potentially fatal autoimmune dermatologic disease. It is characterized by cutaneous damage due to apoptosis of the keratinocytes with consequent dermo-epidermal separation for a $>30 \%$ extension of the body surface, associated with mucosal lesions. It is due to the activation of the immune system, often following the intake of potentially toxic drugs [antibiotics, antiepileptics, non-steroidal antinflammatory drugs (NSAIDs), allopurinol] or after infection with herpetic viruses or mycoplasma. We describe the case of an 82year-old man starting therapy of Allopurinol for hyperuricemia. After four days the patient shows an extensive erythematous rash localized to the trunk and upper limbs. The following day the rash also involves the face, tending to the confluence and after another two days, the macules turn into de-epithelized areas because of dermo-epidermal separation and the lesions involve the oral and ocular mucosa, causing dysphagia and difficulty in speaking. He was treated with steroid and antihistamine therapy, suspending the previously undertaken therapy with antibiotic and Allopurinol.

\section{Introduction}

Toxic epidermal necrolysis (TEN), or Lyell's Syndrome, is a very serious autoimmune dermatologic disease, potentially fatal, with a mortality in elderly patients of about $50 \% .{ }^{1}$ It is a rare condition, with an annual incidence of $0.4-1.9 /$ million. $^{2}$ The primum movens is constituted by the activation of the immune system by a triggering agent (usually a potentially toxic drug). It is estimated that more than 220 drugs are involved as potential causative agents. ${ }^{3}$ In a smaller percentage of cases, however, it is due to infection from herpetic viruses or Mycoplasma. The activation of the immune system determines apoptosis of keratinocytes with development of erythematous lesions and consequent dermo-epidermal separation. The skin lesions are initially manifested as erythematous papules but later develop in typical target or iris lesions with an erythematous periphery and a central zone of necrosis. Macules are initially localized to the trunk and the face and then become de-epithelialized areas for dermoepidermal separation. In $90 \%$ of cases, oral, conjunctival and genital mucosal lesions are present. ${ }^{4}$ Here we describe a case of geriatric patient with a TEN triggered by the intake of Allopurinol.

\section{Case Report}

An 82-years-old man arrived in the Emergency Room for the appearance, for about three days, of dyspnea due to minor efforts. After an initial diagnostic evaluation and a first therapeutic approach, the patient was admitted to a Geriatric ward with a postulated diagnosis of respiratory failure and right pneumonia with basal pleural effusion. The chest radiography showed a right basal pleural opacification and a homolateral mid-basal parenchymal infiltrate. Upon arrival in the ward, the patient appeared lucid, collaborative and apyretic. Vital parameters were normal, $\mathrm{SpO}_{2}$ was $96 \%$ during low-flow oxygen therapy. In pathological anamnesis he reported having hypertensive heart disease, treated with sartans and acetylsalicylic acid. He also reported a hospitalization for a pleural effusion at the age of 68, during wich a non-Hodgkin's lymphoma was diagnosed, subsequently treated, with the complete resolution of the disease. Physical examination confirmed the diagnosis of right basal pleural effusion. Blood chemistry tests showed a rise in white blood cells. An antibiotic therapy was started with Piperacillin/tazobactam $(4+0.5$ g every 8 hours) and Levofloxacin (500 mg a day). On the third day, because the detection of high values of uricemia (10.8 $\mathrm{mg} / \mathrm{dL}$ ), allopurinol $150 \mathrm{mg} /$ day was added to therapy. On the same day, because the increase in pleural effusion, right thoracic drainage was positioned, with the finding of inflammatory exudate, confirmed by histo-
Correspondence: Giuseppe Coppolino, Renal Unit, Department of Health Sciences, Magna Graecia University, Catanzaro; Nephrology and Dialysis Unit, Pugliese-Ciaccio General Hospital, Catanzaro, via Michele Torcia 4, 88100 Catanzaro, Italy.

E-mail: gcoppolino@unicz.it

Key words: Adverse drug reaction; allopurinol; Lyell's syndrome; toxic epidermal necrolysis; elderly.

Received for publication: 30 November 2018. Revision received: 21 March 2019.

Accepted for publication: 29 March 2019

This work is licensed under a Creative Commons Attribution-NonCommercial 4.0 International License (CC BY-NC 4.0).

(C) Copyright R.P. Cerra et al., 2019

Licensee PAGEPress, Italy

Geriatric Care 2019; 5:7958

doi:10.4081/gc.2019.7958

logical examination. After four days, an extensive erythematous rash, with maculopapular lesions, localized to the trunk and upper limbs, was documented (Figure 1). The following day the rash also involved the face, tending to the confluence and after another two days, it extended to the oral and ocular mucosa causing dysphagia and difficulty in speaking. Subsequently, extensive de-epithelialization areas appeared, with a positive Nikolsky sign, elicited with a detachment of the epidermis from lower layers when slightly rubbed. Autoimmune bullous diseases have been excluded from histological examination wich resulted negative for anti-desmoglein antibodies. Serology tests for Herpes Simplex virus and Mycoplasma pneumoniae were performed, with negative results. The cutaneous manifestation suggested a diagnosis of TEN, likely to be attributed to allopurinol, started few days earlier, also considering that the patient was previously tolerant to the antibiotics used. Causality was probable to the drug Allopurinol with a score of seven on Naranjo Causality assessment scale. ${ }^{2}$

The diagnosis was confirmed by the skin biopsy that showed dermo-epidermal separation with suppurative granulomatous folliculitis. The severity score of TEN expressed as SCORTEN (Severity of Illness Score for Toxis Epidermal Necrolysis) was 4 , with a probability of death of $58.3 \%$. Steroid therapy with methylprednisolone 80 $\mathrm{mg}$ /day was initiated by suspending allopurinol and antibiotics, obtaining an initial improvement of the cutaneous manifestations. In the following days, for the detection of anemia $(\mathrm{Hb} 7.2 \mathrm{~g} / \mathrm{dL})$, fever and 
decay of the general conditions, the patient was subjected to blood transfusions and supported by saline-based infusional, parenteral nutritional and antistaminic therapy, achieving, after about two weeks, an improvement of clinical condition, pulmonary disease and renal function. The latter, in fact, had undergone an initial worsening of the same wich occurred in the acute phase of the disease. During the hospitalization, the patient presented an episode of delirium, with an important state of psychomotor agitation. At discharge, after 25 days, there was a general clinical improvement (Figure 2).

\section{Discussion and Conclusions}

TEN is the most serious adverse drug reaction, with a mortality $>50 \% .^{5-7}$ In elderly, co-morbidities and polytherapy are predisposing factors. It is characterized by muco-cutaneous and systemic involvement. If not treated, TEN has serious and fatal complications. This condition differs from the Steven-Johnson Syndrome for the extension of skin lesions which, in case of TEN, is greater than $30 \%$ of the total skin surface. $^{2,6}$ TEN, supported by genetic predisposition, usually affects patients with particular allotypes of human leukocyte antigens. ${ }^{7,8}$ The pathogenesis of this disease is very complex and involves cytotoxic $\mathrm{T}$ lymphocytes, NK cells and other mediators activated by environmental, infectious and pharmacological factors. It can trigger a series of events that lead to keratinocyte apoptosis. ${ }^{8-13}$ Most involved pharmacological classes in TEN are antiepileptics, followed by antibiotics, hypouricemic agents and NSAIDs. ${ }^{11,14,15}$

In our case an adverse reaction to allopurinol was observed. Approximately $5 \%$ of patients who start taking this drug suspend it due to adverse events: ${ }^{16}$ in most cases skin reactions, gastrointestinal and hematological disorders. The risk factors for the occurrence of allopurinol adverse reactions are advanced age, impaired renal function, the presence of the HLA-B*5801 allele, the use of thiazide diuretics, alcoholism and severe liver dysfunction. ${ }^{16-18}$

A recent observational study has documented a stronger association between TEN and allopurinol $(6 / 100,000)$ compared with the association of TEN and COX2 inhibitors and 5 aminosalicylates. The aforementioned study also showed a potential association with proton pump inhibitors, fluoxetine and mirtazapine. ${ }^{19}$

Older age, in particular, as it is characterized by a higher incidence of co-morbidities, polypharmacy, immunosuppression and frailty, correlates significantly with the onset of adverse drug reactions. In the case of our patient, the state of immunosuppression, also linked to the neoplastic pathology, associated

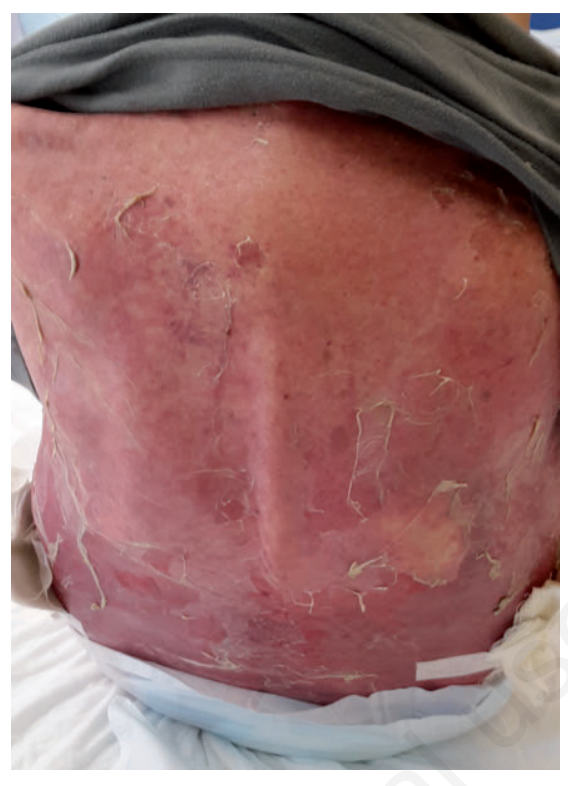

Figure 1. Acute phase.

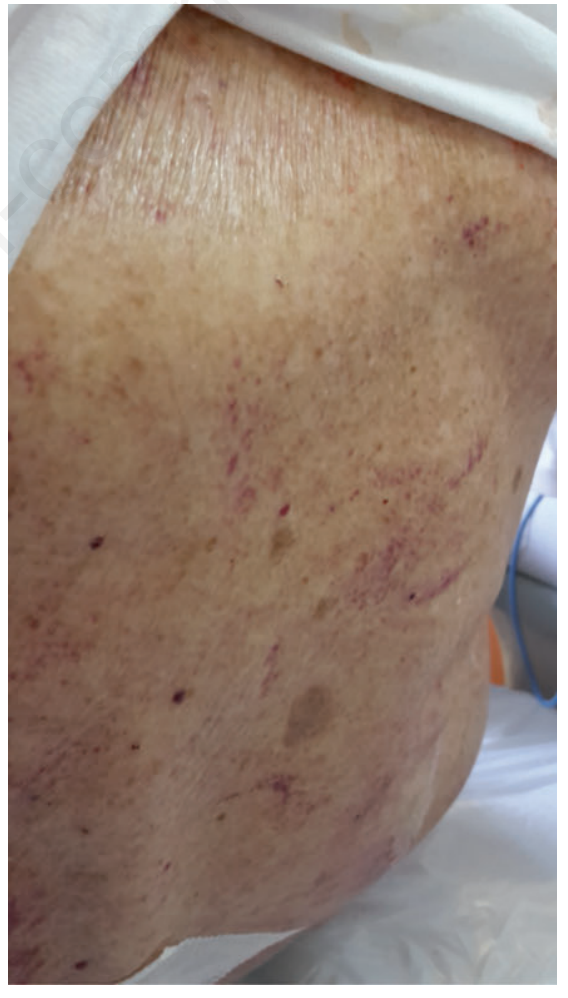

Figure 2. Hospital discharge. with the concomitant conditions and nutritional status, could have constituted a fundamental element in the determinism of this affection. ${ }^{20}$ There is a score that allows estimating the prognosis in these patients, called SCORTEN. The score examines some clinical parameters and makes it possible to formulate a probability of death based on the score obtained, ranging from 0 (mortality of $2.5 \%$ ) to 5 (mortality of $90 \%$ ). ${ }^{21}$ By calculating the SCORTEN score in our patient, we obtained a value of 4 , estimating a probability of death of $58.3 \%$. The therapeutic approach consists in the timely suspension of the triggering drug and in the support of vital functions. ${ }^{1,22,23}$ The prevention of infections is also imperative, with the need for measures of antisepsis, isolation of the patient, intensive and daily skin care.

In conclusion, TEN is a serious disease. Predisposing factors in elderly are co-morbidities and polytherapy. Recognizing the disease in a short time and adopting a personalized therapeutic strategy is important to improve the prognosis and reduce mortality.

\section{References}

1. Abood GJ, Nickoloff BJ, Gamelli RL. Treatment strategies in toxic epidermal necrolysis syndrome: where are we at? J Burn Care Res 2008;29:269-76.

2. Naranjo CA, Busto U, Sellers EM, et al. A method for estimating the probability of adverse drug reactions. Clin Pharmacol Ther 1981;30:239-45.

3. Yang MS, Lee JY, Kim J, et al. Incidence of Stevens-Johnson syndrome and toxic epidermal necrolysis: a nationwide population-based study using national health insurance database in Korea. PLoS One 2016;11:e165933.

4. Harris V, Jackson C, Cooper A. Review of toxic epidermal necrolysis. Int J Mol Sci 2016;17:12.

5. Gerull R, Nelle M, Schaible T. Toxic epidermal necrolysis and StevensJohnson syndrome: a review. Crit Care Med 2011;39:1521-32.

6. Kelly JP, Auquier A, Rzany B, et al. An international collaborative case-control study of severe cutaneous adverse reactions (SCAR). Design and methods. J Clin Epidemiol 1995;48:1099-108.

7. Wang J, Cheng X, Lu Y, Zhou B. A case report of toxic epidermal necrolysis associated with AZD-9291. Drug Design Develop Ther 2018;12:2163-7.

8. Schwartz RA, McDonough PH, Lee BW. Toxic epidermal necrolysis: Part I. Introduction, history, classification, clin- 
ical features, systemic manifestations, etiology, and immunopathogenesis. J Am Acad Dermatol 2013;69:173e1-13; quiz 85-6.

9. Nassif A, Bensussan A, Boumsell L, et al. Toxic epidermal necrolysis: effector cells are drug-specific cytotoxic T cells. J Allergy Clin Immunol 2004;114: 1209-15.

10. Nassif A, Bensussan A, Dorothee G, et al. Drug specific cytotoxic T-cells in the skin lesions of a patient with toxic epidermal necrolysis. J Invest Dermatol 2002;118:728-33.

11. Sethuraman G, Sharma VK, Pahwa P, Khetan P. Causative drugs and clinical outcome in Stevens Johnson syndrome (SJS), toxic epidermal necrolysis (TEN), and SJS-TEN overlap in children. Indian J Dermatol 2012;57:199-200.

12. Coppolino G, Bolignano D, Campo S, et al. Circulating progenitor cells after cold pressor test in hypertensive and uremic patients. Hypertens Res 2008;31:717-24.

13. Buemi M, Senatore M, Gallo GC, et al. Pulmonary hypertension and erythro- poietin. Kidney Blood Press Res 2007; 30:248-52.

14. Roujeau JC, Kelly JP, Naldi L, et al. Medication use and the risk of StevensJohnson syndrome or toxic epidermal necrolysis. N Engl J Med 1995;333: 1600-7.

15. Bastuji-Garin S, Rzany B, Stern RS, et al. Clinical classification of cases of toxic epidermal necrolysis, StevensJohnson syndrome, and erythema multiforme. Archiv Dermatol 1993;129:92-6.

16. Yang DC, Chang CM. Allopurinolinduced drug reaction with eosinophilia and systemic symptoms syndrome with recurrence. J Am Geriatr Soc 2010; 58:2043-4.

17. Stamp LK, Taylor WJ, Jones PB, et al. Starting dose is a risk factor for allopurinol hypersensitivity syndrome: a proposed safe starting dose of allopurinol. Arthrit Rheum 2012;64:2529-36.

18. Visconti L, Leonardi G, Buemi M, et al. Kidney disease and psoriasis: novel evidences beyond old concepts. Clin Rheumatol 2016;35:297-302.
19. Frey N, Bodmer M, Bircher A, et al. Stevens-Johnson syndrome and toxic epidermal necrolysis in association with commonly prescribed drugs in outpatient care other than anti-epileptic drugs and antibiotics: a population-based case-control study. Drug Safety 2018 [Epub ahead of print].

20. Coppolino G, Bolignano D, Gareri P, et al. Kidney function and cognitive decline in frail elderly: two faces of the same coin? Int Urol Nephrol 2018;50: 1505-10.

21. Bastuji-Garin S, Fouchard N, Bertocchi $\mathrm{M}$, et al. SCORTEN: a severity-of-illness score for toxic epidermal necrolysis. J Invest Dermatol 2000;115:149-53.

22. Castelain F, Humbert P. Toxic epidermal necrolysis. Curr Drug Safety 2012; 7:332-8.

23. Schneider JA, Cohen PR. StevensJohnson syndrome and toxic epidermal necrolysis: a concise review with a comprehensive summary of therapeutic interventions emphasizing supportive measures. Adv Ther 2017;34:1235-44. 